\title{
MOLECULAR LINE STUDIES OF DENSE CORE MOTIONS
}

\author{
YUEFANG WU \\ CCAST(World Laboratory), P.O. Box 8730, Beijing 100080 \\ Geophysics Department, Peking University, Beijing 100871
}

\begin{abstract}
Molecular lines have revealed various supporting motions in dense cores. Line widths and emission region sizes of $\mathrm{NH}_{3}$ and $\mathrm{CS}$ in the same kind of cores or of the same line in cores with or without sources are different and can not be explained with the line width- size relationship. Outflows in dense cores show rich characteristics which can account for the $\mathrm{NH}_{3}$ emission difference between the two kinds of the cores; $\mathrm{CS}$ emission is consistent with the chemical effects in shocked regions. Rotation exists in both kinds of cores and may be related to the observed polarities and collimations of outflows.
\end{abstract}

\section{Introduction}

Millimeter lines and infrared studies indicate that small visually opaque regions are the locations of low mass star formation and are usually $0.1-0.3 \mathrm{pc}$ in size, $10^{4}-10^{5} \mathrm{~cm}^{-3}$ in density and have $\mathrm{T}_{\mathrm{k}}$ about 10K (Myers et al. 1983, Myers and Benson 1983, Zhou et al., 1989). About 50\% of these dense cores have associated IRAS sources of which more than half have no optical counterparts and may be the potential protostars(Beichman et.al. ,1986). All of the cores with sources have luminosities $\leq$ $100 \mathrm{~L}_{\theta}$. Cores with and without sources have different properties. In this paper we analyse the inner motions, outflows and rotations, and make comparisons between these two classes of cores.

\section{Core Motions}

Myers et al. (1983) have measured 90 dense cores with the molecular pair ${ }^{13} \mathrm{CO}$ and $\mathrm{C}^{18} \mathrm{O}$. They have compared the sizes, temperatures and densities of the observed cores with the conditions for the equilibrium and stability of a pressure-bounded isothermal sphere, and found that if these cores were supported by turbulent motions and take the typical core with $\Delta \mathrm{v}\left(\mathrm{C}^{18} \mathrm{O}\right)=0.6 \mathrm{kms}^{-1}$ or $\mathrm{T}_{\mathrm{D}}=230 \mathrm{~K}$. equilibrium appears possible and may be stable in this case. It is also consistent with the Larson's model (1981). If the line width partly reflects the supporting motion, many cores are also consistent with turbulent contraction.

With another tracer $\mathrm{NH}_{3}$ and the same method for ${ }^{13} \mathrm{CO}$ and $\mathrm{C}^{18} \mathrm{O}$ data analysis, Myers and Benson(1983) investigated the stability of the cores and found that the equilibrium of these cores may be supported by $10 \mathrm{~K}$ thermal motions plus either a subsonic microturbulence $\left(0.14 \mathrm{kms}^{-1}\right)$ or an early collapse with an age of less than $10^{5} \mathrm{yr}$.

Zhou et a l. (1989) observed CS [ $\mathrm{J}=(2-1),(3-2)$ and (5-4)] line in 27 cores of which 13 have IRAS sources. Comparison between the result and $\mathrm{NH}_{3}$ data shows that the line width differ by a factor of 2 or so. Both the CS lines of $\mathrm{J}=2-1$ and $\mathrm{J}=3$ - 2 are wider than $\mathrm{NH}_{3}$ lines. This situation means that thermal motions can not dominate in the CS regions. The CS line broadening can not be explained with optical depth effect, and it can not be accounted by the emission region size, either. 
On the other hand all the millimeter measurements, indicate that line widths in cores with IRAS sources are wider that those in cores without sources(Table 1).

Table 1 Line Widths and Intensities

\begin{tabular}{|c|c|c|c|c|c|c|}
\hline Core & \multicolumn{3}{|c|}{ Line Width $\left(\mathrm{kms}^{-1}\right)$} & \multicolumn{3}{|c|}{$T_{k}(K)$} \\
\hline Class & $\mathrm{C}^{18} \mathrm{O}^{\mathrm{a}}$ & $\mathrm{NH}_{3}{ }^{\mathrm{d}}$ & $\mathrm{CS}^{\mathrm{b}}$ & $\mathrm{CO}^{\mathrm{c}}$ & $\mathrm{C}^{18} \mathrm{O}^{\mathrm{a}}$ & $\mathrm{NH}_{3}{ }^{\mathrm{d}}$ \\
\hline With & & & & & & \\
\hline $\begin{array}{l}\text { Sources } \\
\text { Without }\end{array}$ & $0.66+0.04$ & $0.42+0.05$ & $1.05+0.06$ & $11.1 \pm 0.2$ & $11.0 \pm 0.6$ & $10.8 \pm 0.4$ \\
\hline Sources & $0.47+0.03$ & $0.27+0.02$ & $0.58+0.04$ & $10.4 \pm 0.9$ & $10.4 \pm 0.6$ & $10.7 \pm 0.5$ \\
\hline
\end{tabular}

Data from: a. Myers et al, 1983. b. Zhou et al, 1989. c. Wu et al, 1990. d. Myers and Benson, 1983

The possible responsible reasons would not be the thermal motion since as Table 1 shows, all $\mathrm{CO}$, $\mathrm{C}^{18} \mathrm{O}$ and $\mathrm{NH}_{3}$ observations indicate the temperatures are nearly the same. Using the relation of the intrinsic line width and the turbulent width: $\Delta \mathrm{v}_{\mathrm{t}}=\left[\left(\Delta \mathrm{v}_{\mathrm{i}}\right)^{2}-8(\ln 2) \mathrm{kT}_{\mathrm{K}} / \mathrm{m}\right]^{1 / 2}$ and the observed data of these molecular species we got $\Delta \mathrm{v}_{\mathrm{t}}$ and size for the two groups of cores, listed in columns $2-7$ of Table 2. These parameters show that the law $\Delta v \alpha R^{1 / 2}$ is broken for all of the molecular line measurements, particularly for $\mathrm{NH}_{3}$ and CS. The correlation of the size-line width has been found from various line-line in the same clouds and the same line for cloud - cloud or region - region. The derived power-law exponents range from $0.3-1.0$ (Myers, 1983). For all the cores of the two groups the relation between line width and size is out of this range. This phenomenon may concern with the role of the young stellar objects.

Table 2 Turbulent Widths and Sizes

\begin{tabular}{|c|c|c|c|c|c|c|}
\hline $\begin{array}{l}\text { Core } \\
\text { Class }\end{array}$ & $\Delta \mathrm{v}_{\mathrm{t}}\left(\mathrm{kms}^{-1}\right)$ & $\begin{array}{l}\mathrm{C}^{18} \mathrm{O} \\
\mathrm{R}(\mathrm{pc})\end{array}$ & $\Delta \mathrm{v}_{\mathrm{t}}\left(\mathrm{kms}^{-1}\right) \mathrm{NH}_{3}$ & $R(p c)$ & $\begin{array}{r}\mathrm{CS} \\
\Delta \mathrm{v}_{\mathrm{t}}\left(\mathrm{kms}^{-1}\right)\end{array}$ & $R(p c)$ \\
\hline $\begin{array}{l}\text { With } \\
\text { Sources } \\
\text { Withoout }\end{array}$ & $0.64+0.05$ & $0.36+0.03$ & $0.41+0.06$ & $0.17+0.03$ & $1.01+0.15$ & $0.34+0.05$ \\
\hline sources & $0.45+0.03$ & $0.31+0.05$ & $0.22+0.02$ & $0.11+0.02$ & $0.55+0.04$ & $0.22+0.07$ \\
\hline
\end{tabular}

\section{CO Outflows}

The outflows in dense cores are usually weaker than those in high mass star formation regions. The energies of these outflows range in $10^{41}-10^{45} \mathrm{erg}, 2$ orders lower than that of high mass sources. The mass loss rates are $10^{-6}-10^{-9} \mathrm{M}_{\theta} \mathrm{yr}^{-1}$, also lower than the high mass ones. Nevertheless, these outflows may have some characteristics. a). They have rather high detected rates(Myers et al.1988, Wu et al. 1990). If we take this kind of outflows within the distance of $500 \mathrm{pc}$, statistics show that the occurence rate is $5.5 \times 10^{-5} / \mathrm{pcc}^{-2} \mathrm{yr}^{-1}$, which may be very close to the star birth rate near the sun(Ostriker et al. 1974, Mezger and Smith, 1977), where $\tau$ is the lifetime of outflows and is about $7 \times 10^{4}$ year on the average(Wu 1990). Their dynamic evolution is rather long: $1 \times 10^{4}-1.9 \times 10^{5}$ yr(Myers et al. 1988, Heyer et al. 1987). The relative long time scale may increase the chance for finding these outflows. b). Morphology: These outflows are more polar-like and less isotropic. All 4 isotropic sources(S140, M8E, NGC 7538 and MWC 1080) are found in the high mass star formation 
regions. Their collimation may be better than that of the high ones(Mao et al. 1989, Wuet al. 1991). c).Optical Phenomena:The most striking feature about low mass outflows is the associated optical phenomenon. HH-objects are thought to be tracers of high velocity gas and to associate with $\mathrm{T}$ Tauri stars almost exclusively. Recently one of the faintest $\mathrm{HH}$ objects was found to be associated with the CO blue peak position in L1582B( Wu et al. 1990). So far over 22 optical jets were found and more than half of them have CO outflows. Stocke et al. (1988) have found that in L1551 the jet $\mathrm{HH}$ objects are bow shock interface between two winds coming from IRS5 region. The second wind has an inferred velocity of about $160 \mathrm{kms}^{-1}$. Molecular outflow results in the momentum flux of this not very high velocity but pervasive wind acting on the material in cores (Mundt, 1988, Stocke et al.1988). d). Roles for the cores: The outflows in dense cores can still put significant momentum and energy in the surrounding gas though they are rather weak. Myers et al. (1988) found that almost in all cases $\mathrm{P}_{\text {flow }}$ is larger than or equal to $\mathrm{P}_{\text {core }}$, and in more than half sources which they analysed, $\mathrm{P}_{\text {flow }} / \mathrm{P}_{\text {core }}=1 \sim 2$ For the line width difference of the two kinds of cores listed in Table 2 , there should be no porblem for $\mathrm{NH}_{3}$ emission regions since $\quad \mathrm{P}_{\text {flow }} \geq \mathrm{P}_{\text {core w. }}>\mathrm{P}_{\text {core w. }}-\mathrm{P}_{\text {core w.o. }}$

For CS regions, theaverage size is a factdr of 1.5 larger than that of the $\mathrm{NH}_{3}$ regions and the CS line width is greater by a factor of 2 . Besides the density is also higher than that of $\mathrm{NH}_{3}$ cores(Zhou et al. 1989). Therefore the momentum of CS regions will be larger than that of $\mathrm{NH}_{3}$ cores by a factor of at least 5 , while $\mathrm{P}_{\text {flow }}$ is only 1 - 2 times of $\mathrm{P}_{\text {core }}$ of the $\mathrm{NH}_{3}$ cores.. Therefore the flow momentum may not be able to enhance the line width in the CS regions. Similar analysis shows that it can not account for the momentum increases of the CS emission regions to the $\mathrm{NH}_{3}$ emission regions, either

For $\mathrm{C}^{18} \mathrm{O}$ emission regions, we calculate the velocity dispersion $\sigma$ are 0.33 and $0.27 \mathrm{kms}^{-1}$ respectively, taking $T_{K}=10 \mathrm{~K}$, for 20 cores with and 23 cores without sources (Myers et al. 1983). The corresponding average core masses are $27.4 \pm 3.7$ and $19.1 \pm 4.0 \mathrm{M}_{\theta}$ respectively. The average momentum difference is $3.9 \mathrm{M}_{\theta} \mathrm{kms}^{-1}$. For 26 low mass outflows (Wu, 1990), the average momentum is $3.3 \mathrm{M}_{\theta} \mathrm{kms}^{-1}$. Thus the outflows could couple momentum to the gas to increase the line widths of the $\mathrm{C}^{18} \mathrm{O}$ emission in cores with sources almost completely. Here the problem about the CS kinetics remains, and it may also exist in the high mass star formation regions.It seems that the gas traced by CS obtains more energy from the stellar winds coming from the center sources. It may be owing to this part of the gas which is located in the inner region bears the brunt when the wind blows out. In L43, the two positions of the maximum CS line widths seem that at these positions the gas is plowing directly into the surrounding gas (Mathieu et al .1988). CS high velocity wings were detected in a number of high mass star fomation regions(Thronson and Lada, 1984, Hayashi et al. 1985). Another effect of the shock is that it heats the gas in post shock regions. According to Hartquist et al. (1980), the reaction $\mathrm{S}+\mathrm{H}_{2} \rightarrow \mathrm{HS}+\mathrm{H}$ could occur at the high temperature of this kind of regions. And consequently, $\mathrm{CS}$ is formed in the presence of $\mathrm{HS}: \mathrm{C}+\mathrm{HS} \rightarrow \mathrm{CS}+\mathrm{H} \quad \mathrm{CS}$ is removed by the reverse reaction of it and by the reactions: $\mathrm{CS}+\mathrm{OH} \rightarrow \mathrm{OCH}+\mathrm{S}$ and $\mathrm{CS}+\mathrm{O} \rightarrow \mathrm{CO}+\mathrm{S}$ Their calculations for the molecular fractional abundances show that the CS abundance increases from $4.7 \times 10^{-12}$ at $3 \times 10^{9} \mathrm{~cm}$ behind the shock to $4.7 \times 10^{-8}$ at a distance of $10^{13} \mathrm{~cm}$, where the CS abundance reaches a plateau of this value since there the abundance of $\mathrm{OH}$ and $\mathrm{O}$ decrease. The velocity of the shock that they considered is $8 \mathrm{kms}^{-1}$. It may be met generally in star forming regions.

\section{Rotation}

Table 3 which is devided into 3 parts lists the rotation parameters. $\Omega$ and $\mathrm{R}_{\text {colll }}$ are quoted from different observations or different authors(Boss, 1987, Clark and Johnson, 1981, Wu et al. 1991). All cores with sources and high mass sources listed in Table III have bipolar outflows. The direction 
Table 3 Rotation and High Velocity Gas

\begin{tabular}{|c|c|c|c|c|c|}
\hline $\begin{array}{l}\text { Source } \\
\text { Name }\end{array}$ & $\begin{array}{c}\Omega \\
\left(10^{-14} \mathrm{rad}^{-1}\right)\end{array}$ & $\begin{array}{l}\text { Polarity } \\
\text { of HVG }\end{array}$ & $\mathrm{R}_{\text {coll }}$ & $\begin{array}{l}\text { Direction of } \\
\text { velocity gradient \& } \\
\text { flow project axis }\end{array}$ & Ref. \\
\hline \multicolumn{6}{|c|}{ Low Mass Cores without Sources } \\
\hline L183 & $5.3,5.6$ & $\begin{array}{l}\text { possible } \\
\text { pedestal }\end{array}$ & & & 1,22 \\
\hline L134N & $2.7,2.9$ & Bluewing & & & 1,2 \\
\hline L1709A & $\sim 10$ & Redwing & & & 2 \\
\hline \multicolumn{6}{|c|}{ Low Mass Cores with Sources } \\
\hline L1455 & $11,8.0$ & Bipolar & 3.7 & $\sim \perp$ & 3,4 \\
\hline L1551 & $25,<6.7$ & Bipolar & 5.2 & Cross Angle $>45^{\circ}$ & $5,6,4$ \\
\hline HH1-2 & $15,<1.2$ & Bipolar & 2.1 & & $3,7,4$ \\
\hline HH26IR & 1.7 & Bipolar & 2.7 & $\perp$ & 8,4 \\
\hline $\mathrm{HH} 24$ & $<0.51$ & Bipolar & 1.0 & & 9,4 \\
\hline L43B & 6.5 & Bipolar & 2.5 & Cross Angle $<45^{\circ}$ & 17 \\
\hline L723 & $10,<6.7$ & Bipolar & 4.1 & $\perp$ & $21,3,4$ \\
\hline L778 & $<2.2$ & Bipolar & 2.3 & & 3 \\
\hline B335 & $2.0,7.0$ & Bipolar & 2.8 & Cross Angle $45^{\circ}$ & $11,3,4$ \\
\hline \multicolumn{6}{|c|}{ High Mass Star Formation Regions } \\
\hline GL490 & $<11$ & Bipolar & 1.9 & & 18,4 \\
\hline GL437 & $<1.1$ & Bipolar & 1.6 & & 3,4 \\
\hline Orion-KL & 40 & Bipolar & 1.0 & $\perp$ & 12,4 \\
\hline NGC2071 & $5.6,13$ & Bipolar & 3.7 & $\perp$ & $13,14,4$ \\
\hline NGC2261 & $<0.83$ & Bipolar & 2.0 & & 19,4 \\
\hline MonR2 & $2.8,15$ & Bipolar & 3.0 & $\sim 1$ & $3,8,4$ \\
\hline G35.2N & 23 & Bipolar & 2.6 & $\perp$ & 15,4 \\
\hline CRL2591 & 5.7 & Bipolar & 1.9 & Cross Angle $>45^{\circ}$ & 20,4 \\
\hline Cep A & $8.9,23$ & Bipolar & $?$ & & $16,10,4$ \\
\hline
\end{tabular}

1 Clark \& Johnson, 1981. 2 Wu et al, 1990. 3 Heyer et al, 1986. 4 Boss, 1987. 5 Kaifu et al, 1984. 6 Batrla \& Menten,1985. 7 Torrelles et al, 1985. 8 Torrelles et al, 1983. 9 Matthews \& Little, 1983. 10 Torrelles et al, 1986a. 11 Menten et al, 1984.12 Hasegawa et al, 1984.13 Bally, 1982.14 Lichten, 1982.15 Little et al, 1985.16 Gusten et al, 1984. 17 Mathieuet al, 1988. 18. Kawabe et al, 1984. 19 Canto et al, 1981. 20 Takano et al, 1986. 21 Torrelles et al, 1986b. 22. Frerking and Langer 1982.

of the gradients of 7 flows among 11 with known directions are perpendicular to the flow axes. It suggests that in most flow sources, the rotation axis is coincided with the flow lobe axis. Column 4 of Table 3 lists the collimation factors which are generally better for the flows with high $\Omega$ than those with low $\Omega$. It is also coincident with the trend that the $R_{\text {coll }}$ is higher for low mass sources than that of high mass ones. These are the important tests of the models suggested by Boss(1987).

Motions in dense cores are related to the forming and the activity of the stars, and the interaction of the stellar objects and the surrounding materials. Observations of the cores at high spatial and spectral resolution is important for latter determination of these processes in the foreseeable future.

The project was supported by NSFC. I thank IAU for partially supporting my presence at IAU Symposium No. 150. 


\section{References}

Bally, J. 1982, Ap. J., 261, 558.

Batrla, W., and Menten, K. M. 1985, Ap. J..(Letters), 298, L19.

Beichman, C. A., Myers, P. C., Emerson, J. P., Harris, S., Mathieu, R., Benson, P. J., and Jennings, R. E. 1986, Ap. J., 307, 337.

Boss, A.P. 1987, Ap. J., 316, 721.

Canto, J., Rodriguez, L. F., Barral, J. F., and Carral, P. 1981, Ap. J., 244, 102.

Clark, F. O., and Johnson, D. R. 1981, Ap. J., 247, 104.

Frerking, M. A., and Langer, W. D. 1982, Ap. J., 256, 523.

Gusten, R., Chini, R., and Neckel, T. 1984, Astr. Ap., 138, 205.

Hartquist, T. W., Oppenheimer, M., and Dalgano, A. 1980, Ap. J., 236, 182.

Hasegawa, T., Kaifu, N., Inatani, J., Morimoto, M., Chikada Y., Hirabayashi, H.,

Iwashita, H., Morita, K., Tojo, A., and Akabane, K. 1984, Ap. J., 283, 117.

Hayashi, M., Omodaka, T., Hasegawa, T., and Suzuki, S. 1985, Ap. J., 288, 170.

Heyer, M. H., Snell, R. L., Goldsmith, P. F., Strom, S. E., and Strom, K. M. 1986,Ap. J., 308, 134.

Heyer, M. H., Snell, R. L., Goldsmith, P. F., and Myers, P. C. 1987, Ap. J., 321, 370.

Kaifu, N., Suzuki, S., Hasegawa, T., Morimoto, M., Inatani, J., Nagane, K., Miyazawa, K.,

Chikada, Y., Kanzawa, T., and Akabane, K. 1984, Astr. Ap., 134, 7.

Kawabe, R., Ogawa, H., Fukui, Y., Takano, T., Takaba, H., Fujimoto, Y., Sugitani, K., and Fujima, M. 1984, Ap. J. (Letters), 282, L73.

Larson, R. B. 1981, M. N. R. A. S., 194, 809.

Lichten, S. M. 1982, Ap. J., 253, 593.

Little, L. T., Dent, W. R. F., Heaton, B., Davies, S. R., White, G. J. 1985, M. N. R. A. S.,.217, 227.

Mao, X., Wu, Y., Hao, J., and Hou, M.1989, Acta.Scientiarum Naturalium, U. Pekinensis, 25,505.

Mathieu, R. D., Bensan, P. J., Fuller, G. A., Myers, P. C., and Schild, R. E. 1988, Ap. J. 330, 385.

Matthews, N., and Little, L.T. 1983, M. N. R. A. S., 205, 123.

Menten, K. M., and Walmsley, C. M. 1985, Astr. Ap., 146, 369.

Mezger, P. G., and Smith, L. F. 1977, in IAU Symposium No. 75, P. 133.

Mundt, R.1988 in Formation and Evolution of Low Mass Stars, ed.A. K.Dupree, M.T. V.Lago,P257 Myers, P. C. 1983, Ap. J., 270, 105.

Myers, P. C., and Benson, P. J.1983, Ap. J., 266, 309.

Myers, P. C., Heyer, M., Snell, R. L., and Goldsmith, P. F. 1988, Ap. J., 324, 907.

Myers, P. C., Linke, R. A., and Benson, P. J. 1983, Ap. J., 264, 517.

Ostriker, J. P., Richstone, D. O., and Thuan, T. X. 1974, Ap. J.(Letters), 188, L87

Stocke, J.T., Hartigan, P. M., Strom, S. E., Strom, K. M., Anderson, E. R.1988,Ap. J. Suppl.,68,279

Takano, T., Stutzki, J., Fukui, Y., and Winnewisser, G. 1986, Astr. Ap., $158,14$.

Thronson, Jr., H. A., and Lada, C. J. 1984, Ap. J., 284, 135.

Torrelles, J. M., Canto, J., Rodriguez, L. F., Ho, P.T.P., Moran, J. M.1985, Ap. J.(Letters) 294,L117

Torrelles, J. M., Ho, P. T. P., Rodriguez, L. F., and Canto, J. 1986a, Ap. J., 305, 721.

Torrelles, J. M., Ho, P. T. P., Moran, J. M., Rodriguez, L. F., and Canto, J. 1986b, Ap. J., 307, 787.

Torrellas, J. M., Rodriguez, L. F., Canto, J., Carral, P., Marcaide, J., Moran, J. M., and

Ho, P. T. P. 1983, Ap. J., 274, 214

Wu, Y. 1990, Progress in astronomy, 8, 291

Wu, Y., Zhou, S., and Evans, N. J. II 1990, in preparation.

Wu, Y., Huang, M., He, J.1991, in The Stellar Populations of Galaxies, ed. by B. Barbuy, in press.

Zhou, S., Wu, Y., Evans, N. J. II, Fuller, G. A., and Myers, P. C. 1989, Ap. J., 346, 168. 


\section{QUESTIONS AND ANSWERS}

A.Leger: If you had no IR data to detect stars, would you really be able to decide whether a cloud is forming star or not from radio data?

Y.Wu: We can consider the line width density which may be rather large or high for the star formation regions; the most strong evidences of the existence of a stellar source from radio observations are bipolar outflow maser line and continuum emissions; we can also see if there is any molecular species overabundant to obtain the representations of the activity of the forming star. Systematic large molecular line velocity shift may also be the evidence of a stellar source under formation. 